\title{
All-trans-retinoic acid inhibits chondrogenesis of rat embryo hindlimb bud mesenchymal cells by downregulating p53 expression
}

\author{
TAO-GEN ZHANG ${ }^{1,2^{*}}$, XUE-DONG LI ${ }^{1,2^{*}}$, GUO-YONG YU ${ }^{1,2^{*}}$, PENG XIE $^{1}$, YUN-GUO WANG $^{1}$, \\ ZHAO-YONG LIU ${ }^{2}$, QUAN HONG ${ }^{2}$, DE-ZHONG LIU ${ }^{2}$ and SHI-XIN DU ${ }^{1}$ \\ ${ }^{1}$ Department of Orthopedics, The Affiliated Luohu Hospital of Shenzhen University, Shenzhen, Guangdong 518000; \\ ${ }^{2}$ Department of Orthopedics, The First Affiliated Hospital, Shantou University Medical College, \\ Shantou, Guangdong 515041, P.R. China
}

Received March 6, 2014; Accepted January 22, 2015

DOI: $10.3892 / \mathrm{mmr} .2015 .3423$

\begin{abstract}
Despite the well-established role of all-trans-retinoic acid (ATRA) in congenital clubfoot (CCF)-like deformities in in vivo models, the essential cellular and molecular targets and the signaling mechanisms for ATRA-induced CCF-like deformities remain to be elucidated. Recent studies have demonstrated that p53 and p21, expressed in the hindlimb bud mesenchyme, regulate cellular proliferation and differentiation, contributing to a significant proportion of embryonic CCF-like abnormalities. The objective of the present study was to investigate the mechanisms for ATRA-induced CCF, by assessing ATRA-regulated chondrogenesis in rat embryo hindlimb bud mesenchymal cells (rEHBMCs) in vitro. The experimental study was based on varying concentrations of ATRA exposure on embryonic day $12.5 \mathrm{rEHBMCs}$ in vitro. The present study demonstrated that ATRA inhibited the proliferation of cells by stimulating apoptotic cell death of rEHBMCs. It was also observed that ATRA induced a dose-dependent reduction of cartilage nodules compared with the control group. Reverse transcription-polymerase chain reaction and western blotting assays revealed that the mRNA and protein expression of cartilage-specific molecules, including aggrecan, Sox 9 and collagen, type II, $\alpha 1$ (Col2a1), were downregulated by ATRA in a dose-dependent manner; the mRNA levels of p53 and p21 were dose-dependently upregulated from 16 to $20 \mathrm{~h}$ of incubation with ATRA, but dose-dependently
\end{abstract}

Correspondence to: Professor Shi-Xin Du, Department of Orthopedics, The Affiliated Luohu Hospital of Shenzhen University, 47 Friendship Road, Shenzhen, Guangdong 518000, P.R. China E-mail: dsx1232013@sina.com

*Contributed equally

Key words: all-trans-retinoic acid, p53, rat embryo hindlimb bud mesenchymal cells, chondrogenesis, in vitro, congenital clubfoot downregulated from 24 to $48 \mathrm{~h}$. Of note, p53 and p21 were regulated at the translational level in parallel with the transcription with rEHBMCs treated with ATRA. Furthermore, the immunofluorescent microscopy assays indicated that proteins of p53 and p21 were predominantly expressed in the cartilage nodules. The present study demonstrated that ATRA decreases the chondrogenesis of rEHBMCs by inhibiting cartilage-specific molecules, including aggrecan, Sox 9 and Col2al, via regulating the expression of $\mathrm{p} 53$ and $\mathrm{p} 21$.

\section{Introduction}

Retinoic acid (RA), an oxidative metabolite of vitamin A, is present in the vertebrate embryonic limb bud and is critical for correct limb development. An excess or a deficiency of all-trans-retinoic acid (ATRA) may cause congenital malformations, including limb defects (1). Delgado-Baeza et al (2) have established a fetal rat clubfoot model using a single intragastric dose of RA on day 10 of gestation. Our group previously demonstrated that inhibition of hindlimb cartilage development is a crucial factor for ATRA-induced congenital clubfoot $(\mathrm{CCF})$ in a rat model $(3,4)$. Limb patterning and growth are carefully regulated through a complex network of transcription factors and signaling molecules $(5,6)$. Despite a well-established role of ATRA in CCF, the essential cellular and molecular targets and the signaling mechanism whereby ATRA induces CCF remain to be elucidated.

A key aspect of embryonic development is dependent upon cell cycle control; p53 is an important mediator of the cell cycle checkpoint at the G1-S transition (7) and is intricately involved in the cellular decision-making process (8). p21, as a cyclin-dependent kinase inhibitor, is a negative regulator of cell cycle progression and is required for chondrocyte differentiation $(9,10)$. Induction of p53 is generally characterized by increased transcription of $\mathrm{p} 21$, whose product interacts with the cyclin-dependent complexes and regulates the cell cycle $(11,12)$. However, it is becoming increasingly clear that p21 can be induced in a p53-independent manner (13). In addition, p53 induces apoptosis or programmed cell death to remove unwanted cells and to prevent teratogenesis in 
preimplantation as well as in the early organogenesis period, which is essential for normal development $(14,15)$.

However, p53-dependent apoptosis is also responsible for excessive cell loss in the predigital regions, which may result in defects of the digits. $p 53^{+/+}$animals have been previously reported to be more sensitive to radiation-induced apoptosis and defects of the digits than $p 53^{+/-}$and $p 53^{-/-}$mice during the mid-gestational period and the late gestational period. However, $p 53^{-/}$embryonic mice were more sensitive to apoptosis and defects than were $p 53^{+/-}$and $p 53^{+/+}$mice during late organogenesis $(16,17)$. As p53-knockout (KO) mice developed and were viable, polydactyly of the hindlimbs and other defects were reported to occur at a higher incidence in p53-deficient mice (18). The importance of p53 may be more pronounced in other species, as p53-KO Xenopus laevis embryos exhibited inhibition of mesodermal differentiation and severe gastrulation defects (19). This difference may be explained by the evidence that other p53 family members, p63 and p73, which are expressed during mouse embryogenesis and may compensate for the absence of p53, are not expressed during early developmental stages in frogs (20). Similar to that observed in the embryonic development of frogs, p53 was revealed to be involved in embryogenesis in zebrafish (21). In addition, inhibiting p53 expression in salamanders resulted in inhibition of limb regeneration (22). High levels of p53 are present in all tissues, including the limb bud until mid-gestation. During organogenesis, p53 levels decrease until they are scarcely detectable in terminally-differentiated tissues (23). However, it remains to be elucidated what specific role p53 has during early embryonic limb bud development.

A synchronized and tightly coupled mechanism is hypothesized to be involved in the two p53-associated processes, with the levels of p53 and cell type being important in bud patterning. The results of the present study supported the possibility that p53 or its signaling pathways may be etiologically responsible for the increased incidence of congenital developmental abnormalities, including CCF. It was hypothesized that ATRA may induce CCF by regulating the expression of p21 during early embryonic development in cartilage-specific molecules, including Sox9, aggrecan and col2al, which are required for chondrogenesis of rat embryo hindlimb bud mesenchymal cells (rEHBMCs). In the present study, mesenchymal cells were collected from embryonic day 12.5 (E12.5) rat embryo hindlimb bud mesenchymal cells (rEHBMCs) and the mechanisms whereby ATRA affected chondrogenesis were investigated in vitro.

\section{Materials and methods}

Animals. Primiparous female Sprague-Dawley (SD) rats, attained from the Laboratory Animal Center of Shantou University Medical College (Shantou, China) were mated with adult males from the same strain and supplier. The day when sperm was detected in the vaginal smear was considered to be day 0 of gestation (E0). Mated females were housed individually in clear polycarbonate cages with stainless steel wire lids and corncob granules as bedding. Food pellets and fitered tap water were available ad libitum. Animals were maintained at $24^{\circ} \mathrm{C}$ with a humidity of $50 \%$ and a 12 -h light/dark cycle. All animal protocols were approved by the Institutional Animal Care and Use Committee of Shantou University Medical College (Shantou, China) and the study was performed in accordance with the established institutional and national guidelines on the experimental use and care of animals.

Cell culture. Cell culturing was performed as described by Flint and Orton (24). Hindlimb bud mesenchymal cells were isolated from E12.5 female SD rats. Briefly, pregnant rats were euthanized with $\mathrm{CO}_{2}$ and embryos from the female rats were separated and placed in calcium- and magnesium-free Earle's balanced salt solution (HyClone Laboratories, Inc., Logan, UT, USA)/fetal bovine serum (Gibco Life Technologies, Carlsbad, CA, USA) (EBSS-CMF/FBS). The distal three-quarters of the subridge at the distal tip of the hindlimbs were dissociated in EBSS-CMF containing 0.1\% trypsin (Gibco Life Technologies), $0.1 \%$ EDTA and $50 \mathrm{mM}$ Tris- $\mathrm{HCl}$ buffer (Shanghai Chemical Reagent Co., Ltd., Shanghai, China) (pH 7.4). All cells were filtered through a pre-moistened $40 \mu \mathrm{m}$ cell strainer to remove the ectoderm and cell clumps, which were rendered into single cell suspensions. Cells were resuspended at a density of $2 \times 10^{7}$ cells $/ \mathrm{ml}$ in $50 \%$ Dulbecco's modified Eagle's medium (DMEM) and 50\% F12 (Gibco Life Technologies) containing 10\% FBS (Gibco Life Technologies), penicillin (100 IU/ml; Sigma-Aldrich, Beijing, China) and streptomycin $(50 \mathrm{mg} / \mathrm{ml}$; Sigma-Aldrich). Cells at a density of $2 \times 10^{7}$ cells $/ \mathrm{ml}$ were plated onto $60-\mathrm{mm}$ dishes with 19 drops of media $(25 \mu \mathrm{l})$ each or a total of $3 \times 10^{5}$ cells in $15 \mathrm{ml}$ media placed as micromass in the center of a 24-well plate. The cells were incubated for $1 \mathrm{~h}$ at $37^{\circ} \mathrm{C}$ under $5 \% \mathrm{CO}_{2}$ to allow attachment prior to being flooded with the above culture medium. The day of cell seeding was referred to as culture day $0(\mathrm{CD} 0)$.

Alcian blue staining. Alcian blue staining of sulfated cartilage glycosaminoglycans was utilized to examine chondrogenic differentiation using a previously described method (25). To demonstrate the deposition of cartilage matrix proteoglycans, representative cultures were collected on day one and two of incubation and stained with $0.5 \%$ alcian blue $8 \mathrm{GX}$ (Sigma-Aldrich, St. Louis, MO, USA), pH 1.0. Alcian blue bound to sulfate was extracted with $6 \mathrm{M}$ guanidine- $\mathrm{HCl}$ and quantified by measuring the absorbance of the extracts at $600 \mathrm{~nm}$ using a microplate reader (Chromate 4300; Awareness Technology, Inc., Palm City, FL, USA). Chondrogenic cartilage was expressed in optical density (OD) value.

Immunofluorescent microscopy. For determination of the distribution of p53 and p21, cells were fixed in ice-cold 4\% $(\mathrm{w} / \mathrm{v})$ paraformaldehyde in phosphate-buffered saline (PBS) for $30 \mathrm{~min}$ and permeabilized with $0.2 \%$ Triton $\mathrm{X}-100$ (Shanghai Chemical Reagent Co., Ltd.) for $10 \mathrm{~min}$ at $40^{\circ} \mathrm{C}$. Cells were treated with $3 \% \mathrm{H}_{2} \mathrm{O}_{2}$ for 20 min and non-specific binding was blocked with $10 \%$ normal goat serum (Wuhan Boster Biological Technology, Ltd., Wuhan, China) for $30 \mathrm{~min}$ at $37^{\circ} \mathrm{C}$. Following washing with PBS three times, cells were incubated with mouse monoclonal anti-p53 (1:200; cat. no. AP062; Beyotime Institute of Biotechnology, Haimen, China) and rabbit polyclonal p21 antibodies (1:200; cat. no. sc-397; Santa Cruz Biotechnology, Dallas, TX, USA) overnight at $4^{\circ} \mathrm{C}$ followed by $1 \mathrm{~h}$ incubation with goat anti-rabbit 
(1:64; cat. no. BA1105; Boster Biological Technology, Ltd.) and goat anti-mouse fluorescein isothiocyanate-conjugated (1:64; cat. no. BA1101; Boster Biological Technology, Ltd.) antibodies. Following counterstaining with DAPI (Invitrogen Life Technologies, Carlsbad, CA, USA), cells were visualized under the Nikon TE2000-S inverted microscope (Nikon, Tokyo, Japan).

Cell proliferation assay. Proliferation of rEHBMCs was determined by the direct counting of cells from micromass cultures. Control and treated cultures were maintained for the indicated periods of time, trypsinized and counted in triplicate using a hemocytometer (Invitrogen Life Technologies).

Flow cytometric analysis. Levels of apoptosis were analyzed using flow cytometry (FACSCalibur; BD Biosciences, Franklin Lakes, NJ, USA). For detection of propidium iodide staining, cells were excited at $488 \mathrm{~nm}$ and emission was detected at $585 \mathrm{~nm}$. The data were analyzed with the use of WinMDI software (v2.9; Bio-soft Net; http://en.bio-soft. net/other/WinMDI.html).

Reverse transcription-polymerase chain reaction (RT-PCR). Total cellular RNA was isolated from mesenchymal cells using an RNA Simple Total RNA kit (Tiangen Biotech, Beijing, China). The concentration of RNA was determined from the optical density of the sample, which was measured at $260 \mathrm{~nm}$. Aliquots of the extracted RNA samples were initially reversely transcribed for first strand cDNA synthesis. The sequences for specific primers (Generay Biotech Co., Shanghai, China) for detecting mRNA transcripts of the rat p53, p21, Sox9, collagen, type II, $\alpha 1$ (Col2al), aggrean and GAPDH genes were as follows: p53 forward, 5'-CCATCTACAAGAAGTCACAACAC-3' and reverse, 5'-CCCAGGACAGGCACAAAC-3'; p21 forward, 5'-TGTCCGTCAGAACCCATG-3' and reverse, 5'-TGGGAAGGTAGAGCTTGG-3'; Sox 9 forward, 5'-TAGCCCTGGTTTCGTTCTCT-3' and reverse, 5'-TCCTGCTCGTCGGTCATCTT-3'; Col2al forward, 5'-GGAGCAGCAAGAGCAAGGAGAAGAA-3' and reverse, 5'-CTCAGTGGACAGTAGACGGAGGAAAGT-3'; aggrecan forward, 5'-GGAGAGGACTGCGTAGTGATGA-3' and reverse, 5'-AGCCTGTGCTTGTAGGTGTTGG-3' and GAPDH forward, 5'-CAGTGCCAGCCTCGTCTCAT-3' and reverse, 5'-AGGGGCCATCCACAGTCTTC-3'. The PCR conditions were as follows: $35^{\circ} \mathrm{C}$ for $15 \mathrm{~min}$, followed by 36 cycles of $95^{\circ} \mathrm{C}$ for $10 \mathrm{sec}$ and $62^{\circ} \mathrm{C}$ for $30 \mathrm{sec}$. The RT-PCR products were resolved using agarose gel electrophoresis. The signal intensity was quantified using Quantity One Software (v4.5.2; Bio-Rad, Hercules, CA, USA). The transcript levels were normalized against GAPDH and the relative mRNA levels were expressed as the ratio of the density of the detected genes to GAPDH at the same time-point.

Western blot analysis. Cellular lysates were prepared using radioimmunoprecipitation assay lysis buffer (Bocai, Shanghai, China). Western blotting assays were performed as described previously (26) and the following antibodies were used for the procedure: rabbit polyclonal anti-p53 (1:1,000; cat. no. 9282; Cell Signaling Technology, Inc., Danvers, MA,
USA), rabbit polyclonal Sox9 (1:2,000; cat. no. ab26414; Abcam, Cambridge, MA, USA), rabbit polyclonal p21 $(1: 1,000$; cat. no. BS6561) and rabbit polyclonal Col2a1 $(1: 1,000$; cat. no. BS1071), and rabbit polyclonal GAPDH (1:1,000; cat. no. BS60630) antibodies (Bioworld Technology, Nanjing, China). The membranes were then incubated with goat anti-rabbit fluorescein isothiocyanate-conjugated secondary antibodies (1:1,000; cat. no. BA1055; Boster Biological Technology, Ltd.). Densitometric analysis was performed using Quantity One Software (v4.5.2; Bio-Rad, Hercules, CA, USA). GAPDH was used as loading control.

Statistical analysis. Values are expressed as the mean \pm standard deviation for three or more independent experiments. Statistical analyses were conducted using SPSS version 17.0 (SPSS Inc., Chicago, IL, USA). Statistical significance was estimated using a one-way analysis of variance with Bonferroni's post-hoc test (multiple comparisons) and the Student-Newman-Keuls test (comparisons between two groups) was used where appropriate. $\mathrm{P}<0.05$ was considered to indicate a statistically significant difference.

\section{Results}

ATRA inhibits chondrogenesis by downregulating cartilage-specific proteins in rEHBMCs. Chondrogenic differentiation is regulated at three stages, which are precartilage condensation, cell proliferation and cartilage nodule formation $(27,28)$. To determine whether ATRA affected chondrogenesis, undifferentiated rEHBMCs were cultured at a density of $2 \times 10^{7}$ cells $/ \mathrm{ml}$ and stimulated with 0 to $10 \mu \mathrm{M}$ ATRA. Precartilage condensation, one of the two main stages of chondrogenesis $(29,30)$, was then assessed using Alcian blue staining for sulfated proteoglycans and cartilage nodules on days one and two. The results were expressed as the ratio of the density of the detected group to that of the control group at the same time-point. It was identified that ATRA caused a marked dose-dependent reduction in the quantity of cartilage $(10 \mu \mathrm{M}$ ATRA, $0.280 \pm 0.035$ vs. controls, $1.200 \pm 0.150 ; \mathrm{P}<0.01)$. At $1 \mu \mathrm{M}$ ATRA, Alcian blue staining uptake was significantly decreased on day two (Fig. 1A and B) and $1 \mu \mathrm{M}$ ATRA was therefore used for all subsequent experiments.

It was further investigated whether ATRA inhibited chondrogenesis by downregulating the expression of cartilage-specific molecules, including aggrecan, Sox 9 and Col2al. The expression of aggrecan, Sox 9 and Col2al on day one was not detected (data not shown). The RT-PCR assays revealed that ATRA dose-dependently reduced the mRNA transcript levels of Sox9, aggrecan and Col2a1 on day two (Fig. 1C and D) with a $40-50 \%$ reduction in the mRNA transcript levels of Sox9, aggrecan and Col2a1 in cells treated with $1 \mu \mathrm{M}$ ATRA. Consistently, a dose-dependent reduction in the protein levels of aggrecan, Sox 9 and Col2al (Fig. 1E and 1F) were also observed. These results demonstrated that ATRA dose-dependently inhibited sulfated proteoglycan accumulation and cartilage nodule formation during chondrogenesis by downregulating cartilage-specific proteins, suggesting that ATRA inhibited chondrogenesis by decreasing precartilage condensation of rEHBMCs. 

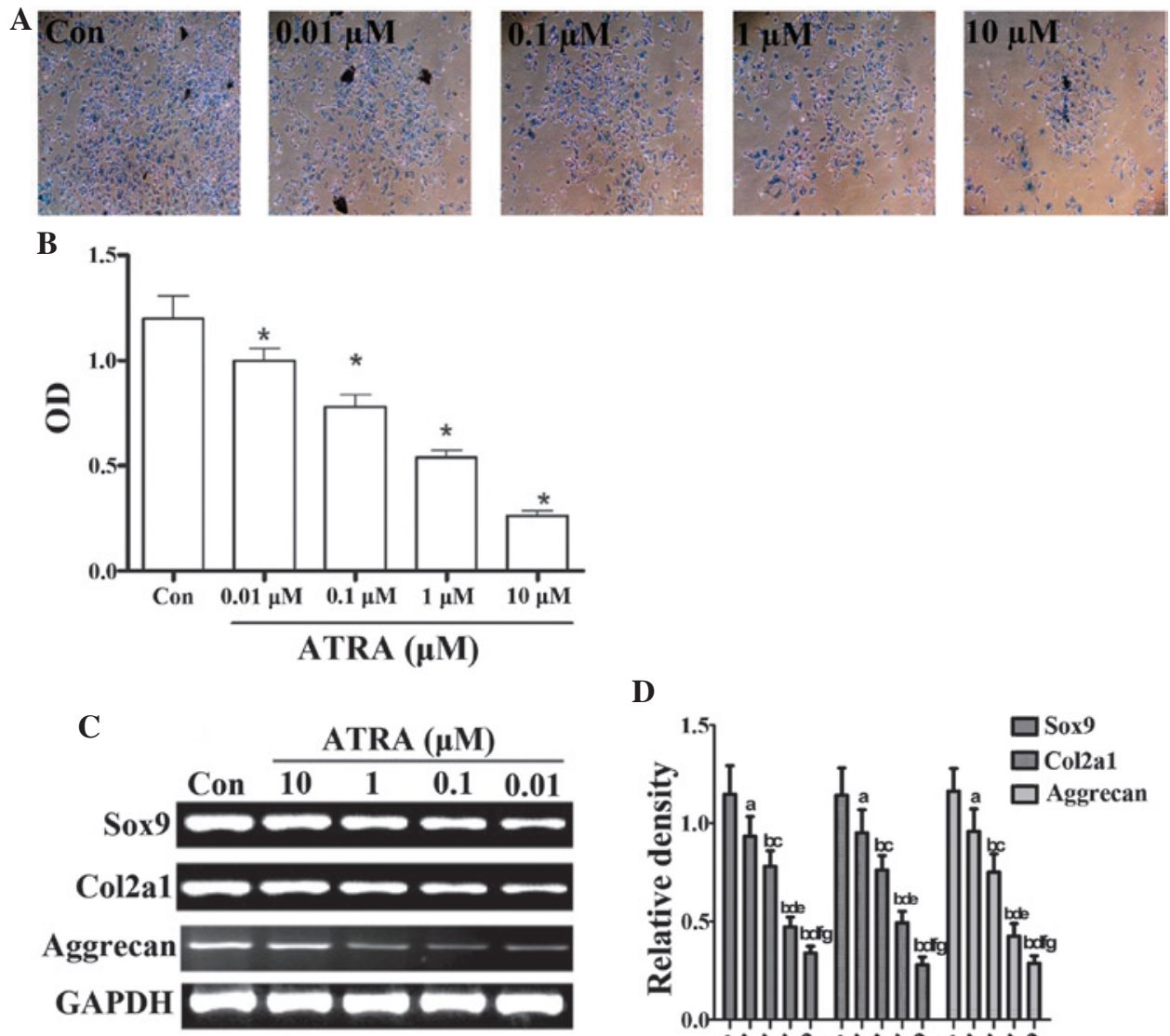

D

$\mathbf{E}$
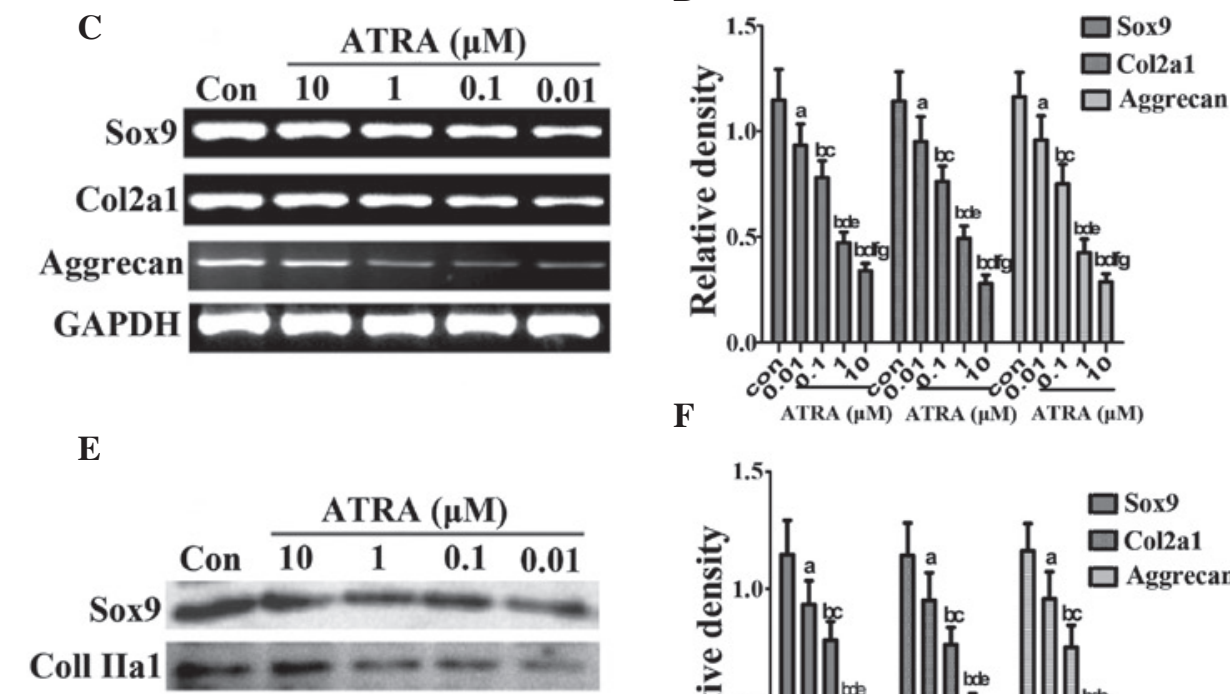

Aggrecan

GAPDH

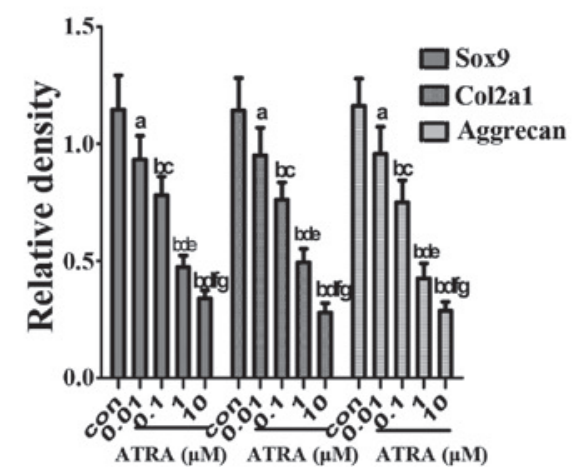

Figure 1. ATRA suppresses chondrogenesis. (A) Alcian blue staining of rEHBMCs grown in micromass cultures in the presence of varying concentrations of ATRA on day two of culture (magnification, x100). (B) Quantification of chondrogenesis was determined by measuring the absorbance of bound Alcian blue at $600 \mathrm{~nm} .{ }^{*} \mathrm{P}<0.05$, compared with control cells. (C) and (E) Changes in the levels of Sox9, aggrecan and Col2a1 in control and ATRA-treated cultures were determined by reverse transcription polymerase chain reaction and western blotting on day two of culture. Results shown are representative of at least three independent experiments. GAPDH was used as a loading control. (D) and (F) Densitometric quantification of Sox9, Col2a1 and aggrecan was performed. (D) ${ }^{\mathrm{a}} \mathrm{P}>0.05$; ${ }^{\mathrm{b}} \mathrm{P}<0.05$, versus control group; ${ }^{\mathrm{C}} \mathrm{P}>0.05$; ${ }^{\mathrm{d}} \mathrm{P}<0.05$, versus $0.01 \mu \mathrm{M}$ ATRA group; ${ }^{\mathrm{e}} \mathrm{P}>0.05$; ${ }^{\mathrm{f}} \mathrm{P}<0.05$, versus $0.1 \mu \mathrm{M}$ ATRA group; ${ }^{\mathrm{g}} \mathrm{P}>0.05$, versus $1 \mu \mathrm{M}$ ATRA group. (F) ${ }^{\mathrm{a}} \mathrm{P}>0.05 ;{ }^{\mathrm{b}} \mathrm{P}<0.05$ versus control group; ${ }^{\mathrm{C}} \mathrm{P}>0.05 ;{ }^{\mathrm{d}} \mathrm{P}<0.05$, versus $0.01 \mu \mathrm{M}$ ATRA group; ${ }^{\mathrm{e}} \mathrm{P}>0.05$; ${ }^{\mathrm{f}} \mathrm{P}<0.05$, versus $0.1 \mu \mathrm{M}$ ATRA group; ${ }^{g} \mathrm{P}>0.05$, versus $1 \mu \mathrm{M}$ ATRA group. ATRA, all-trans-retinoic acid; rEHBMCs, rat embryo hindlimb bud mesenchymal cells; Col2a1, collagen, type II, $\alpha$ 1; OD, optical density.

ATRA stimulates apoptotic cell death of rEHBMCs in vitro. It was subsequently examined whether ATRA-inhibited chondrogenesis occurred as a result of changes in the proliferation of undifferentiated rEHBMCs. It was identified that, compared with the controls, ATRA markedly reduced the viability of rEHBMCs at 6-7 h post-treatment (Fig. 2A). Flow cytometric analysis revealed that ATRA caused a marked increase in the rate of apoptotic cell death of rEHBMCs. At $48 \mathrm{~h}$ post-treatment, the apoptotic rate was $42 \%$ for $\mathrm{rEHBMCs}$ compared with $2 \%$ for the controls $(\mathrm{P}<0.01$; Fig. $2 \mathrm{~B}$ and $\mathrm{C})$.
ATRA modulates p53 and p21 expression in rEHBMCs during chondrogenesis. An important objective of the present study was to elucidate the pattern of p53 expression during differentiation in rEHBMCs. RT-PCR assays revealed that undifferentiated rEHBMCs expressed high mRNA transcription levels of p53. The mRNA transcript levels of p53 markedly increased dose-dependently at 16 and $20 \mathrm{~h}$ after treatment with ATRA, which then underwent a rapid decline 24 and $48 \mathrm{~h}$ after ATRA treatment (Fig. 3A and B). A similar pattern of changes in the levels of p53 
A

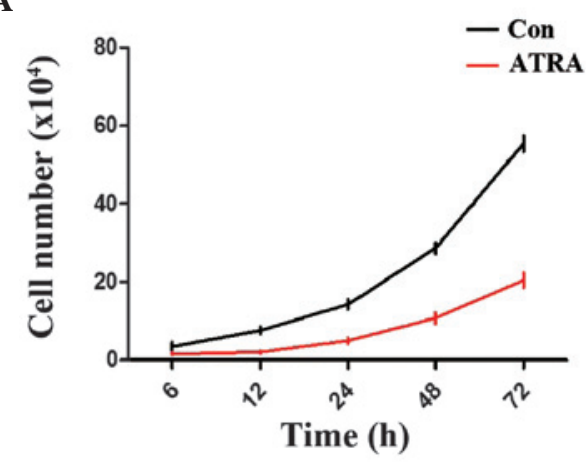

C

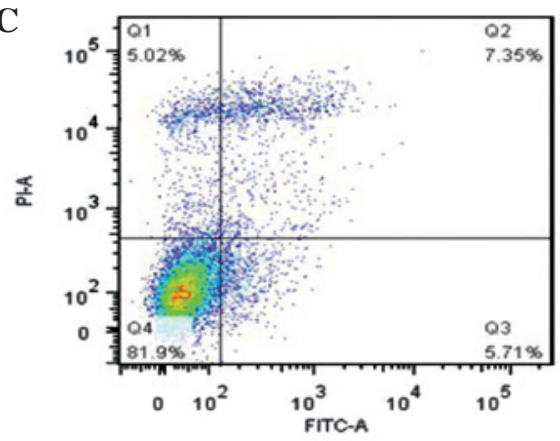

$6 \mathrm{~h}$

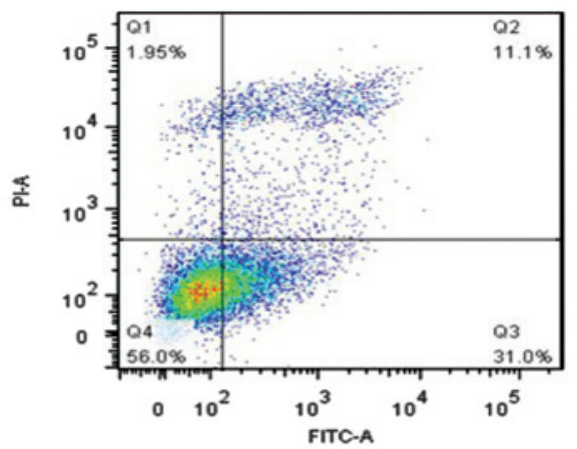

$24 \mathrm{~h}$
B
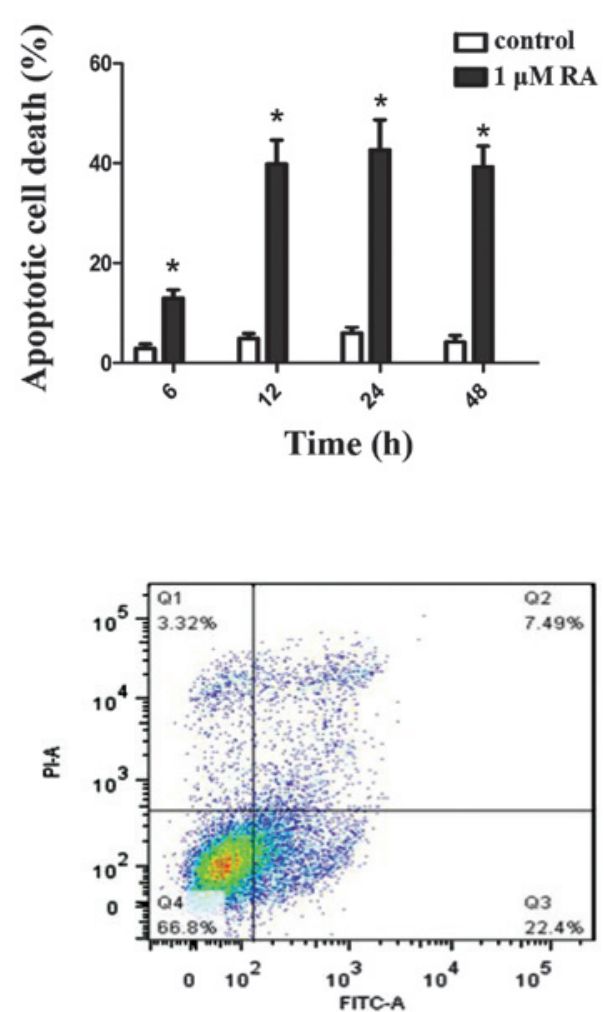

$12 \mathrm{~h}$

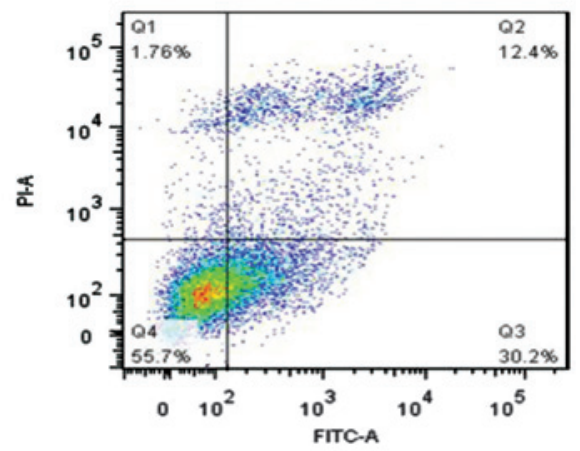

$48 \mathrm{~h}$

Figure 2. ATRA induces apoptotic cell death of chondrogenic competent cells. rEHBMCs were cultured with or without $1 \mu \mathrm{M}$ ATRA. (A) Number of cells was counted at the indicated time-points. (B) Percentages of apoptotic cells were quantified by flow cytometric analysis following $6,12,24$ and $48 \mathrm{~h}$ of culture. Values are expressed as the mean \pm standard deviation of three independent experiments. "P<0.01, compared with control cells. (C) Flow cytometric dot plots at the indicated time-points with Annexin V-FITC and PI double staining for the assessment of the apoptotic rate. ATRA, all-trans-retinoic acid; rEHBMCs, rat embryo hindlimb bud mesenchymal cells; FITC, fluorescein isothiocyanate; PI, propidium iodide.

protein was observed in rEHBMCs following treatment with ATRA (Fig. 3C and D).

p21 inhibits proliferation in vivo and in vitro. Considering the prominent role of p53/p21 in chondrogenesis $(19,20)$, an aim of the present study was to investigate whether ATRA inhibited chondrogenesis of rEHBMCs by modulating the expression of $\mathrm{p} 21$. The expression of p21 was examined between 6 and $48 \mathrm{~h}$ after the induction of chondrogenic differentiation of rEHBMCs. The levels of p21 at 6 and $12 \mathrm{~h}$ remained low or undetectable (data not shown). The levels of p21 markedly increased at 16-20 $\mathrm{h}$ after ATRA treatment; however, they rapidly decreased 24-48 h after treatment (Fig. 4). The immunofluorescent microscopy data further revealed that p53 and p21 were predominantly expressed in the cartilage nodules, mainly in the nucleus (Fig. 5A-D). These findings indicated that ATRA suppressed chondrogenesis of rEHBMCs by modulating the expression of p53/p21 at the transcriptional and translational level.

\section{Discussion}

During early embryonic development, the expression of RA in a specific region of the embryo enables the determination of the position along the embryonic anterior/posterior axis by serving as an intercellular signaling molecule guiding the development of the posterior portion of the embryo (31). Appropriate RA expression in the vertebrate embryo is essential for the proper development and patterning of the organism. It has previously been reported that an excess or a deficiency of RA 
A
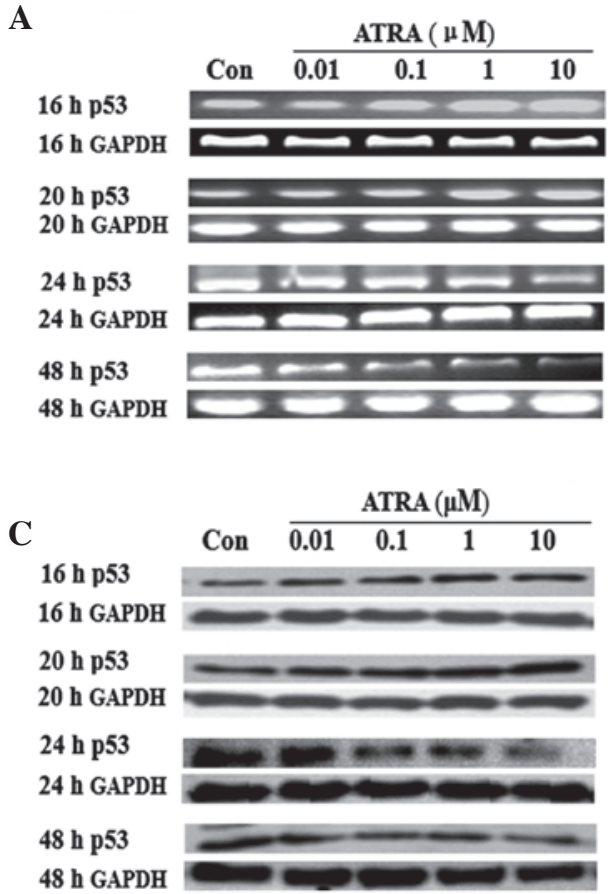

B
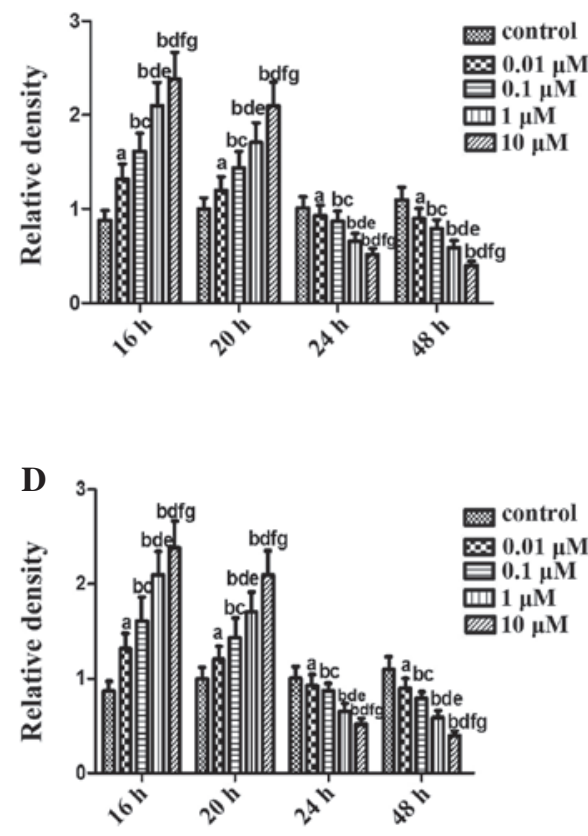

Figure 3. Expression of p53 in spontaneously differentiating rEHBMCs for a period of two days. Undifferentiated mesenchymal cells express high levels of p53 and a decline was noted during differentiation. (A) and (C) Changes in the levels of p53 in control and ATRA-treated cultures were determined by RT-PCR and western blotting at the indicated time-points. Values shown are representative of at least three independent experiments. GAPDH was used as a loading control. (B) and (D) Densitometric quantification of p53 mRNA and p53 protein were performed. (B) p53 mRNA was subjected to RT-PCR in the exponential growth phase and normalized to GAPDH. ${ }^{\mathrm{a}} \mathrm{P}>0.05$, ${ }^{\mathrm{b}} \mathrm{P}<0.05$, versus control group; ${ }^{\mathrm{C}} \mathrm{P}>0.05,{ }^{\mathrm{d}} \mathrm{P}<0.05$, versus $0.01 \mu \mathrm{M}$ ATRA group; ${ }^{\mathrm{e}} \mathrm{P}>0.05$, ${ }^{\mathrm{f}} \mathrm{P}<0.05$, versus $0.1 \mu \mathrm{M}$ ATRA group; ${ }^{\mathrm{P}} \mathrm{P}>0.05$, versus $1 \mu \mathrm{M}$ ATRA group. (D) p53 Protein was subjected to western blot analysis in the exponential growth phase and normalized to GAPDH. ${ }^{a} \mathrm{P}>0.05$; ${ }^{\mathrm{b}} \mathrm{P}<0.05$, versus control group; ${ }^{\mathrm{P}} \mathrm{P}>0.05$; ${ }^{\mathrm{d}} \mathrm{P}<0.05$ versus, $0.01 \mu \mathrm{M}$ ATRA group; ${ }^{\mathrm{e}} \mathrm{P}>0.05$, ${ }^{\mathrm{f}} \mathrm{P}<0.05$, versus $0.1 \mu \mathrm{M}$ ATRA group; ${ }^{\mathrm{g}} \mathrm{P}>0.05$, versus $1 \mu \mathrm{M}$ ATRA group. ATRA, all-trans-retinoic acid; rEHBMCs, rat embryo hindlimb bud mesenchymal cells; RT-PCR, reverse transcription polymerase chain reaction.

A

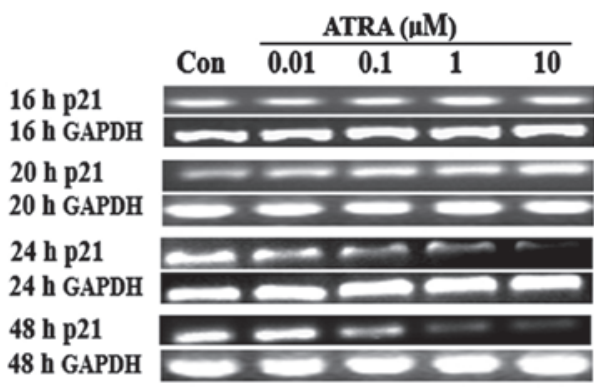

C

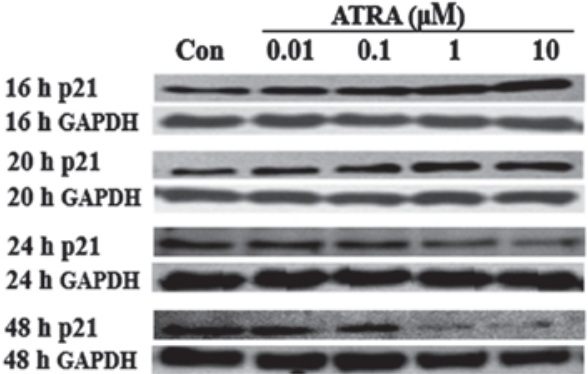

B
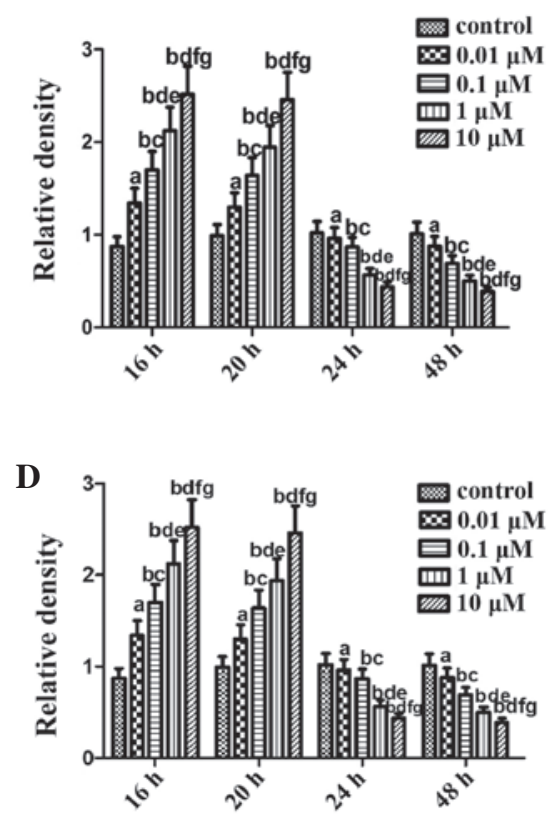

Figure 4. Expression of p21 in spontaneously differentiating rEHBMCs for $48 \mathrm{~h}$. Undifferentiated mesenchymal cells in vitro express high levels of p21 and a decline was noted during differentiation. (A) and (C) Changes in the levels of p21 in control and ATRA-treated cultures were determined by RT-PCR and western blotting at the indicated time-points. Values shown are representative of at least three independent experiments. GAPDH was used as a loading control. (B) and (D) Densitometric quantification of p21 mRNA and p21 protein were performed. (B) p21 mRNA was subjected to RT-PCR in the exponential growth phase and normalized to GAPDH. ${ }^{\mathrm{a}} \mathrm{P}>0.05$; ${ }^{\mathrm{b}} \mathrm{P}<0.05$ versus control group; ${ }^{\mathrm{C}} \mathrm{P}>0.05 ;{ }^{\mathrm{d}} \mathrm{P}<0.05$, versus $0.01 \mu \mathrm{M}$ ATRA group; ${ }^{\mathrm{e}} \mathrm{P}>0.05$; ${ }^{\mathrm{f}} \mathrm{P}<0.05$ versus $0.1 \mu \mathrm{M}$ ATRA group; ${ }^{\mathrm{g} P}>0.05$ versus $1 \mu \mathrm{M}$ ATRA group. (D) $\mathrm{p} 21$ protein was subjected to western blotting in the exponential growth phase and normalized to GAPDH. ${ }^{a} \mathrm{P}>0.05 ;{ }^{b} \mathrm{P}<0.05$, versus control group; ${ }^{\mathrm{C}} \mathrm{P}>0.05 ;{ }^{\mathrm{d}} \mathrm{P}<0.05$, versus $0.01 \mu \mathrm{M}$ ATRA group; ${ }^{\mathrm{e}} \mathrm{P}>0.05 ;{ }^{\mathrm{f}} \mathrm{P}<0.05$ versus $0.1 \mu \mathrm{M}$ ATRA group; ${ }^{\mathrm{g}} \mathrm{P}>0.05$, versus $1 \mu \mathrm{M}$ ATRA group. ATRA, all-trans-retinoic acid; rEHBMCs, rat embryo hindlimb bud mesenchymal cells; RT-PCR, reverse transcription polymerase chain reaction. 

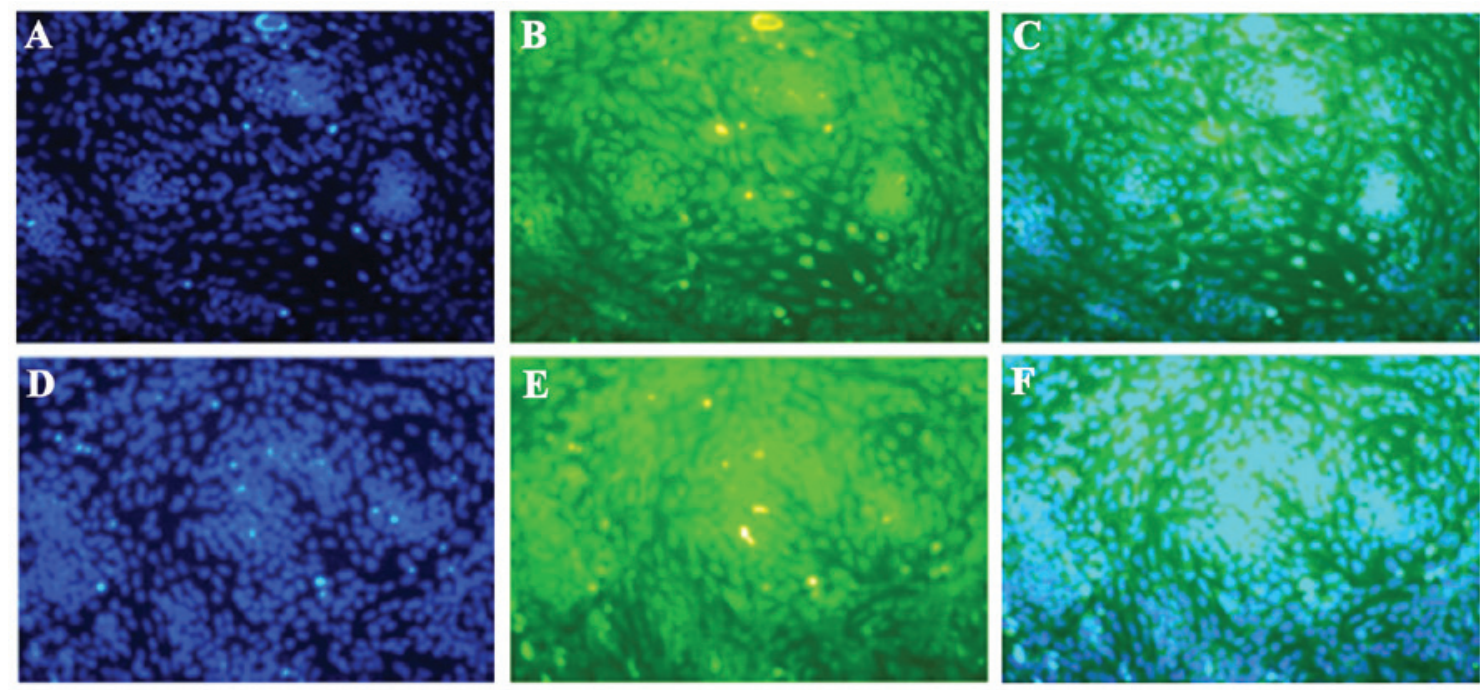

Figure 5. High levels of (B) p53 and (E) p21 in spontaneously differentiating rat embryo hindlimb bud mesenchymal cells in the absence of any inducing agents at $24 \mathrm{~h}$ in vitro. Immunofluorescent microscopy (magnification, $\mathrm{x} 200$ ) revealed that $\mathrm{p} 53$ and p 21 were predominantly expressed in the cartilage nodules, mainly in the nucleus. (A) and (D) were stained with DAPI. (C) is a merged image of (A) and (B) and (F) is a merged image of (D) and (E).

causes congenital malformations, including limb defects (1) that result from the failure of chondrogenesis in the embryonic hindlimb bud mesenchymal cells (32-34). Additionally, it has been proposed that the teratogenic effects of ATRA, leading to limb defects, are based on increased cellular apoptosis in the developing limb $(33,34)$. Previous studies by our group have demonstrated that a rat embryo CCF-like model may be established via a single intragastric dose of ATRA, which exhibits hindlimb congenital malformations, impaired chondrogenesis and increased cellular apoptosis $(3,4,35)$. These findings suggested that defective chondrogenesis of the primary hindlimb bud mesenchymal cells may contribute to CCF.

Formation of cartilage nodules is a morphological characteristic of the condensation stage of chondrogenic development (36). In the present study, it was demonstrated that ATRA dose-dependently reduced the number of cartilage nodules and the area of cartilage nodules in rat hindlimb buds in vitro, suggesting that ATRA inhibited the condensation of hindlimb bud cells. There is a requirement for a minimal number of cells prior to prechondrogenic cells being able to differentiate; if the number of cells is too low, this prevents a skeletal element from forming, which may also reduce the number of skeletal elements formed (37). A reduction in precartilaginous condensations below a critical size is a developmental mechanism for the evolutionary loss of skeletal elements, including chondrocytes. It was identified that ATRA initiated apoptotic cell death of rEHBMCs in the initial $6 \mathrm{~h}$ of ATRA exposure, which decreased the proliferation of rEHBMCs, suggesting that ATRA inhibited the condensation of rEHBMCs by promoting the apoptotic cell death of these cells.

By contrast to the condensation stage, where the formation of cartilage nodules occurs, the differentiation stage is characterized by the expression of a number of specific molecular markers. Sox 9, which is expressed where cartilage formation takes place, is a key transcription factor required for chondrogenesis $(38,39)$. It is essential for mesenchymal condensation prior to chondrogenesis and chondrocyte differentiation fails in the absence of Sox9. Mutations or loss of Sox9 lead to severe malformations of the endochondral skeleton, while homozygous deletion of Sox9 completely inhibits chondrogenesis (40). Of note, Sox9-deficient cells are excluded from cartilage condensation (38), indicating that Sox 9 is required to establish the chondrogenic lineage. Sox9-null embryonic stem cells are inhibited in their differentiation to chondrocytes and persist as mesenchymal cells (38). In the present study, it was identified that ATRA dose-dependently suppressed the expression of Sox9 at the transcriptional and translation level, suggesting that ATRA may inhibit chondrogenesis of the hindlimb bud mesenchymal cells through its inhibitory effect on Sox9.

p21 is required for chondrocytic differentiation $(9,10)$ and its expression is noticeably increased during the differentiation of chondrogenic cells (41). Additionally, p21 is upregulated in maturing and differentiated chondrocytes in vivo (41). Sox9-transfected cells were observed to accumulate in the G0/G1 phase, which was associated with an increase in the expression and promoter activity of p21, suggesting that Sox 9 inhibits cell cycle progression to facilitate its pro-differentiating function. These studies demonstrated that Sox 9 alters the rate of cell cycle progression of chondrocytes and their differentiation by enhancing or inhibiting the expression of p21. It was revealed in the present study that ATRA downregulated the mRNA and protein expression of p21 in primary hindlimb bud mesenchymal cells in a dose-dependent manner. Combined with the evidence that $\mathrm{p} 21$ is predominantly expressed in cartilage cells, these findings indicated that ATRA suppresses chondrogenesis by modulating the expression of Sox 9 and its downstream target $\mathrm{p} 21$ in primary hindlimb bud mesenchymal cells. Col2a1 is the principal constituent of the extracellular cartilage matrix (42). Sox9 has also been observed to be capable of regulating the expression of cartilage-specific molecules, including Col2al and aggrecan $(38,43,44)$. The present study demonstrated that ATRA downregulated the mRNA and protein expression of Col2al and aggrecan in primary hindlimb bud mesenchymal cells in a dose-dependent manner. These findings indicated that ATRA suppresses 
chondrogenesis by modulating the expression of Sox 9 and its downstream target Col2a1 in primary hindlimb bud mesenchymal cells. In addition, the effects occurred early and at a stage prior to the onset of chondrocyte hypertrophy.

Numerous studies have demonstrated that expression of p53 in undifferentiated cells can induce differentiation $(43,45)$. Correspondingly, reduction of p53 expression in human stem cells prevents them from spontaneous apoptosis and differentiation (46). p53 has a central role in cartilage development by regulating key genes for chondrogenesis, including Sox 9 and Col2al (47). It was identified that undifferentiated rEHBMCs express a high level of p53 mRNA and protein. The p53 mRNA transcript levels decreased during early spontaneous differentiation of undifferentiated rEHBMCs in the absence of any inducing agents, which was accompanied by a decrease in p53 protein, suggesting a regulatory mechanism, which is affected not only by protein stability, but also by a decrease in mRNA transcript levels. The levels of p53 were markedly increased from 16-20 $\mathrm{h}$ after treatment with ATRA in a dose-dependent manner. However, the levels then decreased from 24-48 $\mathrm{h}$ after treatment with ATRA in a dose-dependent manner. The results demonstrated that ATRA modulated the expression of p53 in the rat hindlimb bud mesenchymal cells during the entire process of chondrogenesis in a dose- and stage-dependent manner. Of note, ATRA induced apoptosis $10 \mathrm{~h}$ prior to the upregulation of p53. These results demonstrated that ATRA-mediated apoptosis promoted changes in p53, suggesting that p53 upregulation did not initiate ATRA-induced apoptosis during cell proliferation and precartilage condensation of chondrogenic competent cells. ATRA-induced apoptosis of undifferentiated rat hindlimb bud mesenchymal cells may occur independently of p53 function. The levels of p53 were downregulated in parallel with the expression of Sox9, Col2a1 and aggrecan in the primary hindlimb bud mesenchymal cells treated with ATRA. Combined with the evidence that p53 protein is mainly expressed in cartilage cells, these findings suggested that ATRA suppressed the differentiation of undifferentiated rat hindlimb bud mesenchymal cells in association with p53. These findings demonstrated that ATRA inhibited chondrogenesis of primary hindlimb bud mesenchymal cells by downregulating the expression of p53/p21, Sox9, aggrecan and Col2al. Although further investigations are required, it is hypothesized that the effects of ATRA on these chondrogenesis-associated genes may be mediated through modulating the p53/p21 signaling pathways. The p53/p21 signaling pathway may be important for chondrogenesis and the disruption of this process may contribute to a significant proportion of ATRA-induced CCF cases.

\section{Acknowledgments}

The present study was supported by the National Natural Science Foundation of China (grant nos. 81271947, 81341103 and 31240077) and the Administration of Traditional Chinese Medicine of Guangdong Province, China (grant no. 20131248 and 20142084). All experiments were performed at the Laboratory of Molecular Cardiology, the First Affiliated Hospital of Shantou University Medical College (Shantou, China). The authors would like to acknowledge Dr Tao-gen Shang (Xinsteel Centre Hospital, Jiangxi, China) for the time and effort provided to the experiments provided in this study and the support of Professor Yong-gang Zhang, Mr. Li-biao Wu and Mr. Bo-zhi Cai for their helpful advice and collaboration.

\section{References}

1. Lee GS, Kochhar DM and Collins MD: Retinoid-induced limb malformations. Curr Pharm Design 10: 2657-2699, 2004

2. Delgado-Baeza E, Santos-Alvarez I and Martos-Rodriguez A: Retinoic acid-induced clubfoot-like deformity: pathoanatomy in rat fetuses. J Pediatr Orthoped B 8: 12-18, 1999.

3. Zhong YS, Zheng C, Jia Y, et al: Quantitative evaluation in vivo of the degree of differentiation of hindlimb cartilage in a rat clubfoot model. Toxicol Mech Methods 19: 292-297, 2009.

4. Liu ZY,LiXD, Chen B, et al: Retinoic acid retards fetal and hindlimb skeletal development asymmetrically in a retinoic acid-induced clubfoot model. Exp Toxicol Pathol 62: 663-670, 2010.

5. Zelzer E and Olsen BR: The genetic basis for skeletal diseases. Nature 423: 343-348, 2003.

6. Tickle C: Patterning systems - from one end of the limb to the other. Dev Cel 4: 449-458, 2003.

7. Kastan MB, Zhan Q, El-Deiry WS, et al: A mammalian cell cycle checkpoint pathway utilizing p53 and GADD45 is defective in ataxia-telangiectasia. Cell 71: 587-597, 1992.

8. Sarkar SA and Sharma RP: All-trans-retinoic acid-mediated modulation of p53 during neural differentiation in murine embryonic stem cells. Cell Biol Toxicol 18: 243-257, 2002.

9. Beier F, Leask TA, Haque S, et al: Cell cycle genes in chondrocyte proliferation and differentiation. Matrix Biol 18: 109-120, 1999.

10. Beier F, Taylor AC and LuValle P: The Raf-1/MEK/ERK pathway regulates the expression of the $\mathrm{p} 21 \mathrm{Cip} 1 / \mathrm{Waf} 1$ gene in chondrocytes. J Biol Chem 274: 30273-30279, 1999.

11. Wade Harper J, Adami GR, Wei N, Keyomarsi K and Elledge SJ: The p21 Cdk-interacting protein Cip1 is a potent inhibitor of G1 cyclin-dependent kinases. Cell 75: 805-816, 1993.

12. El-Deiry WS, Tokino T, Velculescu VE, et al: WAF1, a potential mediator of p53 tumor suppression. Cell 75: 817-825, 1993.

13. Gartel AL and Tyner AL: Transcriptional regulation of the p21 [(WAF1/CIP1)] gene. Exp Cell Res 246: 280-289, 1999.

14. de Oca Luna RM, Wagner DS and Lozano G: Rescue of early embryonic lethality in mdm2-deficient mice by deletion of p53. Nature 378: 203-206, 1995.

15. Uren AG and Vaux DL: Molecular and clinical aspects of apoptosis. Pharmacol Ther 72: 37-50, 1996.

16. Nomoto S, Ootsuyama A, Shioyama Y, Katsuki M, Kondo S and Norimura T: The high susceptibility of heterozygous p53(+/-) mice to malformation after foetal irradiation is related to sub-competent apoptosis. Int J Radiat Biol 74: 419-429, 1998.

17. Westphal CH, Hoyes KP, Canman CE, et al: Loss of atm radiosensitizes multiple p53 null tissues. Cancer Res 58: 5637-5639, 1998.

18. Armstrong JF, Kaufman MH, Harrison DJ and Clarke AR: High-frequency developmental abnormalities in p53-deficient mice. Curr Biol 5: 931-936, 1995.

19. Cordenonsi M, Dupont S, Maretto S, Insinga A, Imbriano C and Piccolo S: Links between tumor suppressors: p53 is required for TGF- $\beta$ gene responses by cooperating with Smads. Cell 113: 301-314, 2003.

20. Danilova N and Sakamoto KM and Lin S: p53 family in development. Mech Dev 125: 919-931, 2008.

21. Campbell WA, Yang H, Zetterberg $\mathrm{H}$, et al: Zebrafish lacking Alzheimer presenilin enhancer 2 (Pen-2) demonstrate excessive p53-dependent apoptosis and neuronal loss. J Neurochem 96: 1423-1440, 2006.

22. Villiard É, Brinkmann H, Moiseeva O, Mallette FA, Ferbeyre G and Roy S: Urodele p53 tolerates amino acid changes found in p53 variants linked to human cancer. BMC Evol Biol 7: 180, 2007.

23. Schmid P, Lorenz A, Hameister H and Montenarh M: Expression of p53 during mouse embryogenesis. Development 113: 857-865, 1991.

24. Flint $\mathrm{O}$ and Orton $\mathrm{T}$ : An in vitro assay for teratogens with cultures of rat embryo midbrain and limb bud cells. Toxicol Appl Pharmacol 76: 383-395, 1984.

25. Lev R and Spicer S: Specific staining of sulphate groups with alcian blue at low pH. J Histochem Cytochem 12: 309-309, 1964.

26. Li Y, Jiang Y, Wan Y, et al: Medroxyprogestogen enhances apoptosis of SKOV-3 cells via inhibition of the PI3K/Akt signaling pathway. J Biomed Res 27: 43-50, 2013. 
27. Solursh M: Differentiation of cartilage and bone. Curr Opin Cell Biol 1: 989-994, 1989.

28. Ruberte E, Dolle P, Chambon P and Morriss-Kay G: Retinoic acid receptors and cellular retinoid binding proteins. II. Their differential pattern of transcription during early morphogenesis in mouse embryos. Development 111: 45-60, 1991.

29. Thorogood P and Hinchliffe J: An analysis of the condensation process during chondrogenesis in the embryonic chick hindlimb. J Embryol Exp Morphol 33: 581-606, 1975.

30. Ahrens PB, Solursh M and Reiter RS: Stage-related capacity for limb chondrogenesis in cell culture. Dev Biol 60: 69-82, 1977.

31. Duester G: Retinoic acid synthesis and signaling during early organogenesis. Cell 134: 921-931, 2008.

32. Galdones E and Hales BF: Retinoic acid receptor gamma-induced misregulation of chondrogenesis in the murine limb bud in vitro. Toxicol Sci 106: 223-232, 2008.

33. Kochlar D: Cellular basis of congenital limb deformity induced in mice by vitamin A. Birth Defects Orig Artic Ser 13: 111-154, 1977.

34. Paulsen DF, Chen WD, Pang L, Johnson B and Okello D: Stageand region-dependent chondrogenesis and growth of chick wing-bud mesenchyme in serum-containing and defined tissue culture media. Dev Dyn 200: 39-52, 1994.

35. Jia YL, Zhong YS, Chen B, et al: The primary research of posterior buds in quantificated congenital clubfoot at pathological and molecule levels. Chin J Exp Surg 6: 726-728, 2009.

36. Hall BK and Miyake T: All for one and one for all: condensations and the initiation of skeletal development. Bioessays 22 : $138-147,2000$

37. Hall B and Miyake T: The membranous skeleton: the role of cell condensations in vertebrate skeletogenesis. Anat Embryol (Berl) 186: 107-124, 1992.
38. Bi W, Deng JM, Zhang Z, Behringer RR and de Crombrugghe B: Sox 9 is required for cartilage formation. Nat Genet 22: 85-89, 1999 .

39. Bi W, Huang W, Whitworth DJ, et al: Haploinsufficiency of Sox9 results in defective cartilage primordia and premature skeletal mineralization. Proc Natl Acad Sci USA 98: 6698-6703, 2001.

40. Goldring MB, Tsuchimochi $\mathrm{K}$ and Ijiri K: The control of chondrogenesis. J Cell Biochem 97: 33-44, 2006.

41. Stewart M, Farnum C and MacLeod J: Expression of p21CIP1/WAF1 in chondrocytes. Calcif Tissue Int 61: 199-204, 1997.

42. de Crombrugghe B, Lefebvre V and Nakashima K: Regulatory mechanisms in the pathways of cartilage and bone formation. Curr Opin Cell Biol 13: 721-727, 2001.

43. Almog N and Rotter V: Involvement of p53 in cell differentiation and development. Biochim Biophys Acta 1333: F1-F27, 1997.

44. Ng LJ, Wheatley S, Muscat GE, et al: SOX9 binds DNA, activates transcription and coexpresses with type II collagen during chondrogenesis in the mouse. Dev Biol 183: 108-121, 1997.

45. Meletis K, Wirta V, Hede S-M, Nistér M, Lundeberg J and Frisén J: p53 suppresses the self-renewal of adult neural stem cells. Development 133: 363-369, 2006.

46. Qin H, Yu T, Qing T, et al: Regulation of apoptosis and differentiation by p53 in human embryonic stem cells. J Biol Chem 282: 5842-5852, 2007.

47. Ikeda T, Kawaguchi H, Saito T, et al: p63 plays a central role in cartilage development by directly regulating key genes for chondrogenesis. J Bone Miner Res 22: s52-s101, 2007. 